\title{
Orthogonal Complex Hadamard Spreading Codes for I/Q Imbalance Mitigation in MC-CDMA Systems
}

\author{
Jens P. Elsner*, Piotr Rykaczewski ${ }^{\dagger}$, Christian Körner* and Friedrich K. Jondral* \\ * Universität Karlsruhe (TH), Germany \\ \{elsner, koerner, fj\}@int.uni-karlsruhe.de \\ $\dagger$ A.T. Kearney Warsaw Office, Poland \\ Communications \& High Tech Practice \\ piotr.rykaczewski@atkearney.com \\ (on leave from Universität Karlsruhe (TH), Germany)
}

\begin{abstract}
Zero-IF (direct conversion) receivers are very sensitive to non-linearities of analog hardware. A phase and/or amplitude imbalance between the inphase and quadrature signal branch results in a base band signal deterioration known as $I / Q$ imbalance. Especially OFDM and the OFDM-based MC-CDMA modulation technique are affected by this error. This paper proposes a novel orthogonal quaternary MC-CDMA spreading code scheme that uses the inherent mathematical symmetry to mitigate I/Q imbalance without additional computational cost.
\end{abstract}

\section{INTRODUCTION}

In MC-CDMA systems orthogonal spreading codes are used to spread user symbols in the frequency domain. MC-CDMA is usually proposed for synchronous downlink scenarios. In a synchronous multi-user environment orthogonality can easily be restored using low-complexity equalization techniques such as orthogonality restoring combining (ORC), equal gain combining or MMSE combining. Accordingly, the following considerations are based on a MC-CDMA downlink scenario with I/Q imbalance due to non-ideal quadrature demodulation in the receiver. [1] raised the question about the performance of different spreading code sets under I/Q imbalance. This question will be considered here for the MC-CDMA case.

The paper is structured as follows: Section II derives the system model of I/Q imbalance in MC-CDMA systems in fading environments. Section III states basic requirements for spreading codes in MC-CDMA systems and investigates the influence of I/Q imbalance. In section IV new quaternary codes exhibiting good properties with respect to I/Q imbalance are proposed. Section V gives numerical verification through Monte Carlo simulations, followed by a concluding discussion in section VI.

\section{I/Q IMBALANCE ModeL}

I/Q imbalance has been studied in various contexts, cf. [2], [3], [1], [4]. The analytical model used in this paper was adopted from [1] and modified accordingly. A non-ideal local oscillator is described by:

$$
x_{\mathrm{LO}}(t)=K_{1} e^{-j \omega_{\mathrm{LO}} t}+K_{2} e^{j \omega_{\mathrm{LO}} t}
$$

The constants $K_{1}$ and $K_{2}$ are functions of a given phase error $\phi$ and amplitude imbalance $\epsilon$ :

$$
\begin{aligned}
& K_{1}=\frac{(1-\epsilon) e^{j \frac{\phi}{2}}+(1+\epsilon) e^{-j \frac{\phi}{2}}}{2} \\
& K_{2}=\frac{(1-\epsilon) e^{-j \frac{\phi}{2}}-(1+\epsilon) e^{j \frac{\phi}{2}}}{2}
\end{aligned}
$$

The image rejection ratio (IRR) is then given by:

$$
\operatorname{IRR}=\left|\frac{K_{1}}{K_{2}}\right|^{2}
$$

This model assumes frequency-flat I/Q imbalance and absence of a carrier frequency offset.

\section{A. Single Carrier Modulation}

If a single carrier signal with equivalent complex base band signal $z(t)$ is demodulated using the non-ideal oscillator given by (1), the imbalanced base band signal $x(t)$ results in the receiver:

$$
x(t)=K_{1} z(t)+K_{2} z^{*}(t)
$$

The frequency domain representation is:

$$
X(f)=K_{1} Z(f)+K_{2} Z^{*}(-f)
$$

As can be seen from these equations, I/Q imbalance results in weighted self-interference. This is depicted in Fig. 1.

\section{B. OFDM}

If OFDM modulation is used, the subcarriers on mirror frequencies interfere with each other. The DC carrier is typically not used in OFDM systems. Since it also simplifies the mathematical analysis, the DC carrier is considered unused in this paper. The symbols $Z_{n}$ to be transmitted in one OFDM symbol in the frequency domain are given by 


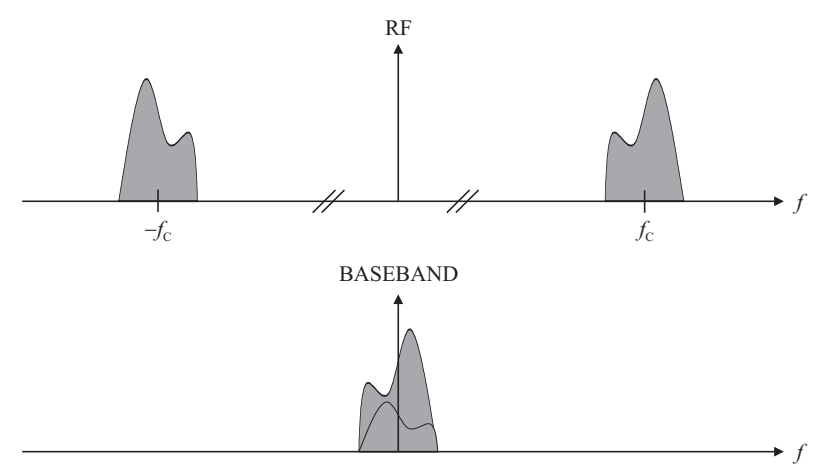

Fig. 1. I/Q imbalance as self-interference

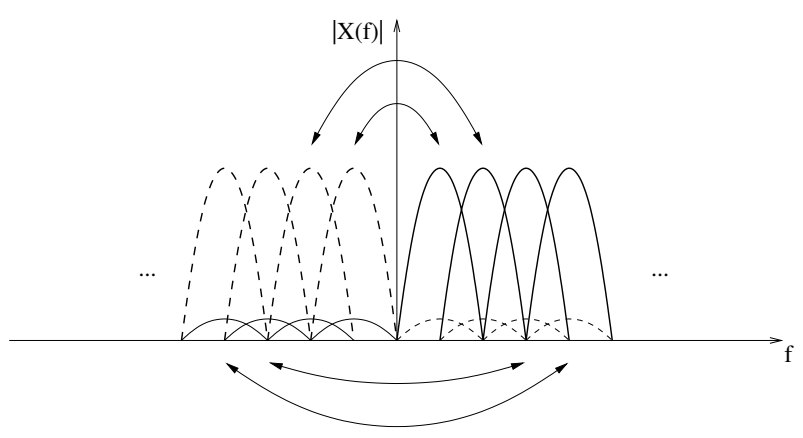

Fig. 2. I/Q imbalance in $\mathrm{MC}$ systems as mutual subcarrier interference in the base band

$\vec{Z}=\left(Z_{-\frac{N}{2}}, \ldots, Z_{-1}, 0, Z_{1}, \ldots, Z_{\frac{N}{2}}\right)^{T}$ where $\mathrm{N}$ is the (even) number of subcarriers. The unused DC carrier is denoted by a zero. The cylic prefix usually used in OFDM systems does not change the frequency domain properties and can hence be neglected here. In the absence of intersymbol (ISI) and intercarrier interference (ICI) induced by other sources than $\mathrm{I} / \mathrm{Q}$ imbalance, the following model for $\mathrm{I} / \mathrm{Q}$ imbalance in OFDM systems results:

$$
X(f)=K_{1} Z(f)+K_{2} \tilde{Z}(f)^{*}
$$

$Z \tilde{(f)})^{*}$ denotes the inverted and conjugated vector $\left(Z_{\frac{N}{2}}^{*}, \ldots, Z_{1}^{*}, 0, Z_{-1}^{*}, \ldots, Z_{-\frac{N}{2}}^{*}\right)$. This interference of subcarriers is depicted in Fig. 2.

\section{C. $M C$-CDMA}

The OFDM model can easily be adopted for MC-CDMA. The MC-CDMA downlink described here has a maximum number of $n$ users, which equals the spreading code length. The number of subcarriers actually used depends on the underlying OFDM system (see Section III-B). The users are separated in the code domain by different spreading codes $C=\left(\overrightarrow{c_{0}}, \ldots, \overrightarrow{c_{n-1}}\right)$ of length $n$. Since the spreading codes are orthogonal, $C^{H} C=n I$ holds (assuming $\left|C_{i j}\right|=1$ ). The symbols sent to different users are written as a column vector $\vec{z}=\left(z_{0}, z_{1}, \ldots, z_{n-1}\right)^{T}$. The resulting chip sequence is given by $C \vec{z}$. This chip sequence is then transmitted over the underlying OFDM subsystem. In the following discussion, the channel noise has been omitted for clarity.
1) I/Q imbalance, frequency-flat fading: (8) describes the resulting imbalanced symbols after non-ideal demodulation and despreading. The matrix $D$ stands for the zero-padded (unused DC carrier) IDFT. It has the property $D^{-1} D^{*}=\tilde{I} . \tilde{I}$ denotes the flipped identity matrix.

$$
\begin{aligned}
\vec{x} & =K_{1} \vec{z}+K_{2} C^{H} D^{-1} D^{*} C^{*} \vec{z}^{*} \\
& =K_{1} \vec{z}+K_{2} C^{H} \tilde{I} C^{*} \vec{z}^{*} \\
& =K_{1} \vec{z}+K_{2} C^{H} \tilde{C}^{*} \vec{z}^{*}
\end{aligned}
$$

Again, $\tilde{C}^{*}$ describes the inverted and conjugated spreading code matrix $\tilde{C}=\left(\tilde{\vec{c}}_{0}^{*}, \ldots, \tilde{\vec{K}}_{-1}^{*}\right)$. It follows from (8) that I/Q imbalance results in multiple access interference (MAI), which properties are described by $C^{H} \tilde{C}$. This matrix describes the correlation between spreading codes and the time-inverted and conjugates spreading codes. Frequency-flat fading results in the attenuation of all subcarriers, given by a multiplication with a random fading coefficient $\mathbf{h}$. This leads to the following model:

$$
\vec{x}=\mathbf{h} K_{1} \vec{z}+\mathbf{h}^{*} K_{2} C^{H} \tilde{C}^{*} \vec{z}^{*}
$$

2) I/Q imbalance, frequency-selective fading: $H$ is a square matrix which models the Rayleigh fading channel in the frequency domain. In the absence of ISI, $H$ is diagonal. The Rayleigh distributed fading coefficients are the diagonal elements, denoted by random variables $\mathbf{h}_{\mathbf{i}}$.

$$
\operatorname{diag}(H)=\left(\mathbf{h}_{\mathbf{1}}, \mathbf{h}_{\mathbf{2}}, \mathbf{h}_{\mathbf{3}}, \ldots, \mathbf{h}_{\mathbf{n}}\right)
$$

With an equalizer matrix $G$ (e.g. $G=H^{-1}$ for ORC) we arrive at the following model:

$$
\vec{x}=K_{1} C^{H} D^{-1} G H D C \vec{z}+K_{2} C^{H} D^{-1} G H^{*} D^{*} C^{*} \vec{z}^{*}
$$

This describes the I/Q imbalance model in a MC-CDMA system with a frequency domain channel $H$. The I/Q-MAI matrix is now given by $C^{H} D^{-1} G H^{*} D^{*} C^{*}$. The corresponding interference profile can be described by calculating the expected value $\mathrm{E}\left\{\left|C^{H} D^{-1} G H^{*} D^{*} C^{*}\right|\right\}$, where the absolute value is to be taken over the elements of the matrix. Hence, the I/Q-MAI depends on spreading mechanism, channel and equalizer approach. The interfering symbol is distorted by the conjugated channel.

\section{COMPlex SPREAding CODES FOR MC-CDMA DOWNLINK}

As shown mathematically, I/Q imbalance leads to MAI. This I/Q-MAI depends on the spreading code properties, as described by $C^{H} \tilde{C}$ in the case of frequency flat fading in (9). Conventionally, real Walsh-Hadamard codes are used as spreading codes. In general, the following criteria have to be considered when searching for a good MC-CDMA spreading code set:

- orthogonality of column (row) vectors, $C^{H} C=n I$,

- equal spreading of information to all subcarriers (cf. [5]), meaning equal magnitude of all entries in the spreading code matrix (without loss of generality $\left|C_{i j}\right|=1$ ),

- PAPR performance (cf. [6], [5]), 
- low complexity of the spreading operation,

- and additionally, considered here, performance under I/Q imbalance.

It is important to note that even mathematically equivalent Hadamard matrices (in the case of real Hadamard matrices: row or column permutation, multiplication of rows or columns by \pm 1 ) exhibit different properties with regard to these criteria. Only matrices which can be derived from each other by column permutation are equivalent with respect to multi-user communication.

\section{A. Minimizing I/Q-MAI}

(8) will be used as a basis for our analysis. As the I/QMAI magnitude measured by the Frobenius norm is always the same in one given orthogonal spreading code set $C$, the goal can only be to minimize the effects of I/Q imbalance over the system load.

$$
\left\|C^{H} \tilde{C}^{*}\right\|_{F}=n
$$

(12) describes this property of the I/Q-MAI, the proof can be found in the appendix. One remaining degree of freedom is the distribution of the I/Q-MAI. Minimum I/Q-MAI between two given spreading codes $\vec{c}_{1}$ and $\vec{c}_{2}$ means that these spreading codes are orthogonal to their time inverted and conjugated counterparts and to each other, $\overrightarrow{c_{1}} \tilde{\vec{c}}_{1}^{*}=0, \overrightarrow{c_{1}} \tilde{\vec{c}}_{2}^{*}=0, \overrightarrow{c_{2}} \tilde{\vec{c}}_{2}^{*}=$ $0, \overrightarrow{c_{2}} \tilde{\vec{c}}_{1}^{*}=0$. For any given load, an active user in the system should interfere with a minimum of other active users in the system. This leads to the definition of an I/Q-optimal spreading code set $C$ :

$$
\left|C^{H} \tilde{C}^{*}\right|=n \tilde{I}
$$

An active user interferes only with one other user and not with oneself. This way, if no other distortion is present, I/Q-MAI can be mitigated up to half load.

1) Frequency flat fading: Frequency flat fading over all subcarriers does not disturb the code symmetry in the frequency domain. The channel matrix can then be written as $H=\mathbf{h} I$ which leads to (assumed here ORC with $G=H^{-1}, K_{1}$ treated as channel influence) the following model:

$$
\hat{\vec{z}}=\vec{z}+K_{2} \frac{\mathbf{h}^{*}}{\mathbf{h}} C^{H} \tilde{C}^{*} \vec{z}^{*}
$$

As can be seen from (14), I/Q-optimal spreading codes offer an advantage in flat fading scenarios. If a spreading code set satisfies (13), I/Q-MAI can be totally avoided up to half load. E.g., the Hadamard matrix

$$
C_{I / Q}=\left(\begin{array}{cccc}
1 & 1 & 1 & -1 \\
1 & 1 & -1 & 1 \\
1 & -1 & 1 & 1 \\
-1 & 1 & 1 & 1
\end{array}\right)
$$

is orthogonal $\left(C^{H} C=n I\right)$ and I/Q-optimal since

$$
C_{I / Q}^{H} C_{I / Q}{ }^{*}=\left(\begin{array}{cccc}
0 & 0 & 0 & 4 \\
0 & 0 & 4 & 0 \\
0 & 4 & 0 & 0 \\
4 & 0 & 0 & 0
\end{array}\right) .
$$

As can be seen from the I/Q-MAI matrix, user 1 and 2 do not interfere with each other or themselves. If the system is filled in the correct order, I/Q imbalance cancels itself up to half load (here: 2 users).

2) Frequency selective fading: Frequency selective fading destroys the code orthogonality and I/Q optimality. The equalizer restores orthogonality to a certain extent but has an almost unpredictable effect on the I/Q-MAI. As simulations indicate, in Rayleigh fading channels all orthogonal codes seem to perform alike with respect to I/Q imbalance. However, an AWGN (or flat fading) channel can be regarded as a Rician fading channel with infinite Rice factor (strength of lineof-sight component). The direct path profits from the code structure and does not result in additional I/Q-MAI. Section $\mathrm{V}$ delivers simulation results supporting this thesis.

\section{B. Frequency interleaving}

To improve frequency diversity, frequency interleavers are used to separate correlated chips in the frequency domain. Frequency interleaving has to be taken into account when designing spreading codes as it affects the frequency code symmetry. A frequency interleaver can be described as a permutation $\pi$ of the chip vector $C \vec{z}$ with a corresponding real permutation matrix $P_{\pi}$. The inverse permutation matrix is then $P_{\pi^{-1}}=P_{\pi}^{-1}$. Hence, the I/Q-MAI model is given by (17).

$$
\vec{x}=K_{1} \vec{z}+K_{2} C^{H} P_{\pi}^{-1} D^{-1} D^{*} P_{\pi}^{*} C^{*} \vec{z}^{*} .
$$

The I/Q-optimality criterion for a code matrix $C$ and a frequency interleaver $P_{\pi}$ is then:

$$
\left|C^{H} P_{\pi}^{-1} \tilde{P}_{\pi}^{*} C^{*}\right|=n \tilde{I}
$$

If we use an I/Q-optimal code set as defined in (13), (18) is satisfied when $\tilde{I} P_{\pi}=P_{\pi} \tilde{I}$. This means that the interleaver matrix has to be rotationally symmetric. E.g., a standard square block interleaver given by (19) (row-wise writing, columnwise reading) is rotationally symmetric.

$$
\left[\begin{array}{cccc}
1 & 2 & \ldots & n \\
1+n & 2+n & \ldots & 2 n \\
\ldots & \ldots & \ldots & \ldots \\
1+(n-1) n & 2+(n-1) n & \ldots & n^{2}
\end{array}\right]
$$

For other sizes, the interleaver has to be designed accordingly. Further research is required here. See [7] for lower bounds on the existence and construction of block interleavers.

\section{USE OF COMPLEX HADAMARD MATRICES IN COMMUNICATION SYSTEMS}

A complex Hadamard matrix $C$ of size $n$ is defined as a square matrix with unimodular entries of arbitrary phase and mutually orthogonal columns $\left(C^{H} C=n I\right)$. An important example is the scaled Fourier matrix. [8] contains an introduction and a list of the known complex Hadamard matrices. A subset of the complex Hadamard matrices are the Butson-type Hadamard matrices [9]. A Butson-type Hadamard matrix $H(q, n)$ of size $n$ is a square matrix whose entries are powers of the $q$-th root of unity. $H(2, n)$ is then the set of 
the well-known real Hadamard matrices. Complex Hadamard matrices can be used as orthogonal spreading codes in MCCDMA systems. However, the complexity of the algorithm used for the linear transform has to be considered in a practical implementation.

\section{A. Examples of I/Q-optimal quaternary complex Hadamard} codes

In [10], the use of Butson-type complex Hadamard matrices $H(4, n)$ was proposed for use in MC-CDMA systems. Compared to $H(2, n)$ Hadamard matrices, the additional degree of freedom offers potentially better PAPR and correlation properties. The matrix entries $H_{i j} \in\{1,-1, j,-j\}$ allow for efficient spreading without multiplication. [10] used a $H(4,2)$ matrix with three free parameters as a starting point to construct $H\left(4,2^{k}\right)$ matrices using the Kronecker construction. The construction is given here for reference:

$$
\begin{gathered}
U_{n}=\bigotimes_{i=1}^{n} U_{1}=U_{1} \otimes U_{n-1}=U_{1} \otimes \cdots \otimes U_{1} \\
U_{1}=\left(\begin{array}{cc}
\mu_{1} & \mu_{1} \mu_{3} \\
\mu_{2} & -\mu_{2} \mu_{3}
\end{array}\right)
\end{gathered}
$$

This set contains the standard $H(2, n)$ Hadamard matrices as the special case $\mu_{1}=\mu_{2}=\mu_{3}=1$. Of these $H\left(4,2^{k}\right)$ matrices, none are I/Q-optimal. There are however I/Q-optimal quaternary codes related to these codes of order 4.

\section{B. Examples of I/Q-optimal Butson-type Hadamard matrices} $H(4,4)$

Since MC-CDMA spreading codes are typically short, all $H(4,4)$ matrices have been evaluated with respect to I/Q imbalance in a computer search in [11]. 2048 of them are I/Q-optimal and non-equivalent in a multi-user context. They can be constructed by

$$
U_{i q_{1}}=\left(\begin{array}{cccc}
-\mu_{1} \mu_{5} & \mu_{2} \mu_{5} & \mu_{3} \mu_{5} & -\mu_{4} \mu_{5} \\
-\mu_{1} & \mu_{2} & -\mu_{3} & \mu_{4} \\
-\mu_{1} \mu_{5} & -\mu_{2} \mu_{5} & \mu_{3} \mu_{5} & \mu_{4} \mu_{5} \\
\mu_{1} & \mu_{2} & \mu_{3} & \mu_{4}
\end{array}\right)
$$

and

$$
U_{i q_{2}}=\left(\begin{array}{cccc}
-j \mu_{3} \mu_{4} & j \mu_{3} \mu_{5} & -j \mu_{1} \mu_{3} & j \mu_{1} \mu_{3} \mu_{4} \\
\mu_{2} \mu_{4} & \mu_{2} \mu_{5} & \mu_{1} \mu_{2} & \mu_{1} \mu_{2} \mu_{4} \\
-\mu_{3} \mu_{4} & \mu_{3} \mu_{5} & \mu_{1} \mu_{3} & -\mu_{1} \mu_{3} \mu_{4} \\
j \mu_{2} \mu_{4} & j \mu_{2} \mu_{5} & -j \mu_{1} \mu_{2} & -j \mu_{1} \mu_{2} \mu_{4}
\end{array}\right)
$$

with $\mu_{1}, \mu_{2}, \mu_{3}, \mu_{4}, \mu_{5}, \in\{1,-1, j,-j\}$.

\section{Construction of higher order I/Q-optimal codes}

To construct I/Q-optimal spreading codes of higher order, rotationally symmetric I/Q-optimal codes $(C \tilde{I}=\tilde{I} C)$ can be used. The Kronecker product of such a matrix with itself is again rotational symmetric and I/Q-optimal (24, proof in appendix).

$$
(C \otimes C)^{H}(\widetilde{\widetilde{\otimes} C})^{*}=n^{2} \tilde{I}
$$

64 out of the $2048 H(4,4)$ I/Q-optimal matrices introduced are rotationally symmetric [11].

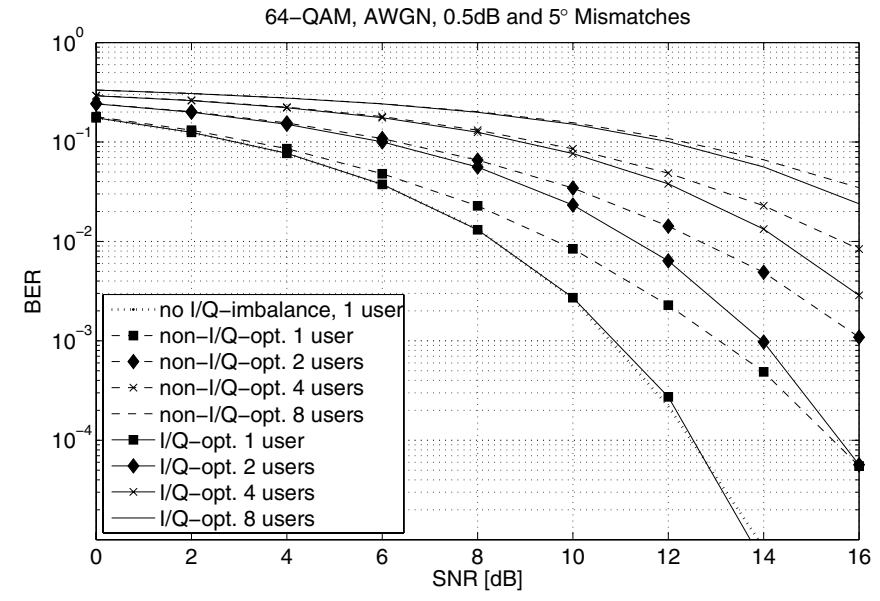

Fig. 3. Comparison of non-I/Q-optimal and I/Q-optimal Hadamard codes in the AWGN channel

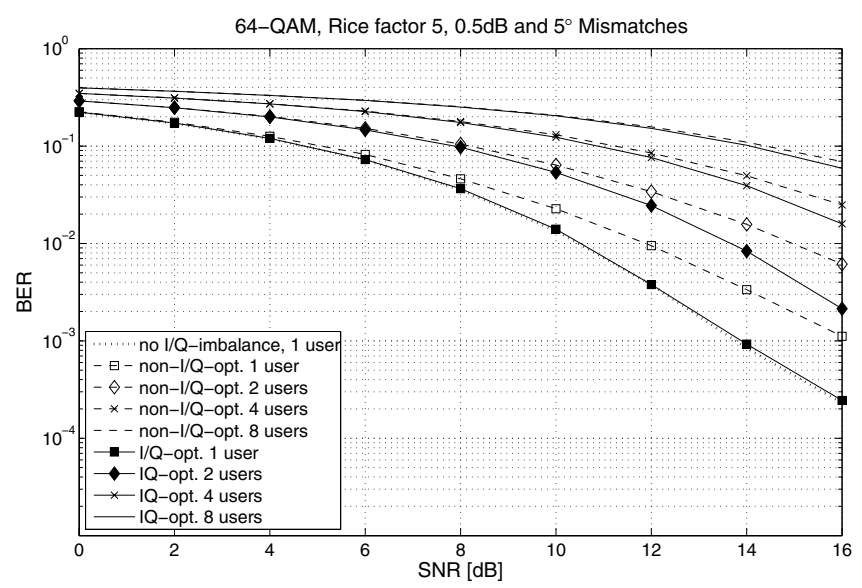

Fig. 4. Comparison of non-I/Q-optimal and I/Q-optimal Hadamard codes, rice-fading, AWGN

\section{Numerical Results}

Fig. 3 shows an MC-CDMA system up to half load with a non-I/Q-optimal $H(2,16)$ standard Hadamard code and an $H(4,16)$ I/Q-optimal complex Hadamard code. Hence the spreading code length is 16 , equaling the maximum number of users. Each user uses a 64-QAM signal constellation. As can be seen from the figure, the $H(4,16)$ I/Q-optimal code performs better for any load. I/Q imbalance is eliminated due to the code structure.

Fig. 4 shows the same MC-CDMA system, this time in a Rice fading scenario with a Rice factor (the ratio of the signal power in the dominant path to the power of the scattered paths) of 5. The I/Q-optimal code still performs better.

Fig. 5 compares the performance of an I/Q-optimal and nonoptimal Hadamard spreading code depending on the strength of the LOS path. As can be seen from the figure, the error floor resulting from I/Q-MAI is significantly lowered by using I/Q-optimal codes. 


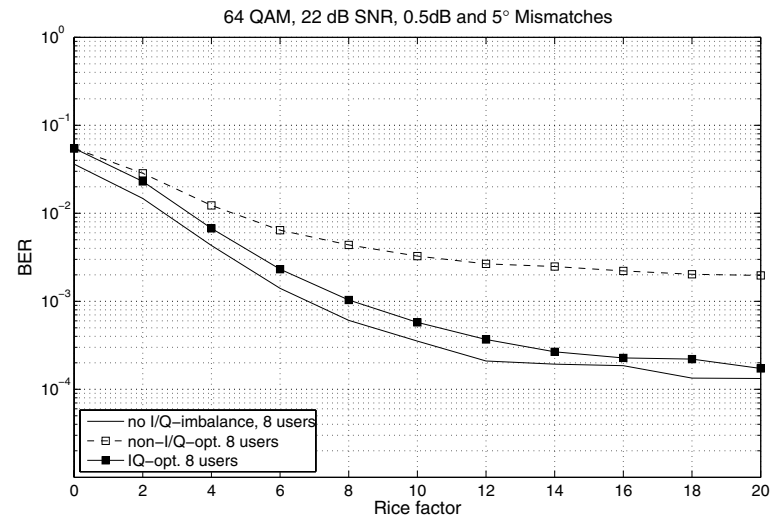

Fig. 5. BER over Rice factor, standard MMSE combining

\section{CONCLUSion AND Future WORK}

This paper establishes the connection between spreading codes in MC-CDMA systems and performance under I/Q imbalance. It was shown that various orthogonal spreading codes exhibit different performance under I/Q imbalance. Orthogonal spreading codes with good properties with respect to I/Q imbalance and an allocation scheme with optimal performance were proposed. The proposed scheme offers maximum gain over other codes in scenarios with frequency flat fading. Since the effect is based on mathematical symmetry, using I/Q-optimal codes results in a performance gain without additional computational costs or loss of frequency diversity. $\mathrm{I} / \mathrm{Q}$ imbalance is a key problem in high speed (16-QAM an above) MC-CDMA direct conversion systems, especially when using low-cost direct-conversion transceivers. Optimizing the spreading codes in this respect has to be considered in addition to compensating the effects in the base band (cf. [12]). An example of I/Q-optimal spreading codes has been given to demonstrate the performance gain. Future work will examine all known Butson-type Hadamard matrices with respect to I/Q imbalance and other criteria given in section III. Furthermore, a stochastic model for I/Q imbalance in MC-CDMA systems incorporating the influence of spreading codes will be developed. [13] and [14] are a first steps towards this goal.

\section{APPENDIX PROOFS}

Proof: (13). Let $C$ be a complex Hadamard matrix of size $n \times n$. For the Frobenius norm $\|A\|_{F}=\sqrt{\operatorname{Tr}\left(A A^{H}\right)}$ holds. The matrix trace satisfies $\operatorname{Tr}(A B)=\operatorname{Tr}(B A)$ and $(A B)^{H}=$ $B^{H} A^{H}$. Hence:

$$
\begin{aligned}
\left\|C^{H} \tilde{C}^{*}\right\|_{F} & =\sqrt{\operatorname{Tr}\left(C^{H} \tilde{C}^{*}\left(C^{H} \tilde{C}^{*}\right)^{H}\right)} \\
& =\sqrt{\operatorname{Tr}\left(C^{H} \tilde{C}^{*}\left(\tilde{C}^{*}\right)^{H} C\right)} \\
& =\sqrt{\operatorname{Tr}\left(C^{H} C\left(\tilde{C}^{*}\right)^{H} \tilde{C}^{*}\right)} \\
& =\sqrt{\operatorname{Tr}(n I n I)} \\
& =\sqrt{\operatorname{Tr}\left(n^{2} I\right)}=n
\end{aligned}
$$

Proof: (24). Let $C$ be a rotationally symmetric $(C \tilde{I}=$ $\tilde{I} C$ ) complex Hadamard matrix. Then, by using the properties of the Kronecker product:

$$
\begin{aligned}
(C \otimes C)^{H} \tilde{I}(C \otimes C)^{*} & =n^{2} \tilde{I} \\
\Leftrightarrow(C \otimes C)^{H}(C \otimes C)^{*} & =n^{2} I \\
\Leftrightarrow\left(C^{H} \otimes C^{H}\right)\left(C^{*} \otimes C^{*}\right) & =n^{2} I \\
\Leftrightarrow\left(C^{H} C^{*}\right) \otimes\left(C^{H} C^{*}\right) & =n^{2} I \\
\Leftrightarrow n I \otimes n I & =n^{2} I
\end{aligned}
$$

\section{REFERENCES}

[1] P. Rykaczewski, M. Valkama, and M. Renfors, "Analytical Approach to I/Q Imbalance in OFDM, CDMA and MC-CDMA Based Systems," in Proc. IEEE Radio and Wireless Symposium (RWS), San Diego, CA, USA, Jan. 2006, pp. 555-558.

[2] M. Valkama, "Advanced I/Q Signal Processing for Wideband Receivers: Models and Algorithms," Ph.D. dissertation, Tampereen teknillinen yliopisto. Julkaisu - Tampere University of Technology. Publication 350.

[3] P. Rykaczewski, "Quadraturempfänger für Software Defined Radios: Kompensation von Gleichlauffehlern," Ph.D. dissertation, Forschungsberichte aus dem Institut für Nachrichtentechnik der Universität Karlsruhe (TH), Band 15, 2006, (In German).

[4] P. Rykaczewski, D. Pieńkowski, R. Circa, and B. Steinke, "Signal Path Optimization in Software Defined Radio Systems," IEEE Trans. Microwave Theory and Techniques, Special Issue on Multifunctional RF Systems, vol. 53, no. 3, pp. 1056-1064, Mar. 2005.

[5] A. Bury, J. Egle, and J. Egle, "Diversity Comparison of Spreading Transforms for Multicarrier Spread Spectrum Transmision," IEEE Trans. on Communications, vol. 51, no. 5, pp. 774-781, May 2003.

[6] E. Pogossova, K. Egiazarian, and J. Astola, "Spreading Sequences For Downlink MC-CDMA Transmission," in Proc. 60th IEEE Vehicular Technology Conference, vol. 7, Sept. 2004, pp. 4859-4863.

[7] V. Tarokh and B. Hochwald, "Existence and Construction of Block Interleavers," in IEEE International Conference on Communications, vol. 3, 2002, pp. 1855-1857.

[8] W. Tadej and K. Zyczkowski, "A Consise Guide to Complex Hadamard Matrices," Open Systems and Information Dynamics, vol. 13, pp. 133177, 2006, Springer Netherlands.

[9] A. T. Butson, "Generalized Hadamard Matrices," in Proc. American Mathematical Society, vol. 13, 1962, pp. 894-898.

[10] Z. Gu, X. Shoulie, and S. Rahardja, "Unified Complex Hadamard Transform Sequences for Multi-carrier CDMA Systems," in Proc. 59th IEEE Vehicular Technology Conference, vol. 3, May 2004, pp. 15141517.

[11] J. P. Elsner, "In Bezug auf I/Q-Fehler optimale orthogonale komplexe Spreizkodes für den MC-CDMA Downlink," 2006, Institut für Nachrichtentechnik, Universitität Karlsruhe, B.Sc. thesis, In German.

[12] P. Rykaczewski, M. Valkama, M. Renfors, and F. Jondral, "Non-DataAided I/Q-Imbalance Compensation Using Measured Receiver FrontEnd Signals," in Proc. IEEE International Symposium on Personal, Indoor and Mobile Communications, Helsinki, Finland, Sept. 2006.

[13] M. Windisch and G. Fettweis, "Performance Degradation due to I/Q Imbalance in Multi-Carrier Direct Conversion Receivers: A Theoretical Analysis," in Proc. IEEE International Conference on Communications, June 2006.

[14] — " "On the Impact of I/Q Imbalance in Multi- Carrier Systems for Different Channel Scenarios," in Proc. IEEE International Symposium on Circuits and Systems, May 2007, invited paper, accepted for publication. 